\title{
Neurodegeneration and energy metabolism: from chemistry to clinics
}

\author{
F Blandini ${ }^{1}$, KH Braunewell ${ }^{2}$, M Manahan-Vaughan ${ }^{3}, \mathrm{~F}$ Orzi $^{4}$ and P Sarti ${ }^{*, 5}$ \\ ${ }^{1}$ Laboratory of Functional Neurochemistry, Neurological Institute 'C. Mondino', Pavia, Italy \\ 2 Signal Transduction Research Group, Neuroscience Research Center of the Charite/Institute for Physiology Humboldt University Berlin, Tucholskystr. 2, Berlin \\ D-10117, Germany \\ ${ }^{3}$ Learning and Memory Research, International Graduate School of Neuroscience, Ruhr University Bochum, FNO 1/116, Universitaetsstr 150, Bochum 44780, \\ Germany \\ 4 Department of Neurological Sciences, University of Rome 'La Sapienza', Rome I-00185, Italy \\ 5 Department of Biochemical Sciences, University of Rome 'La Sapienza', Rome I-00185, Italy \\ All authors have contributed to this work equally \\ * Corresponding author: P Sarti, Dipartimento di Scienze Biochimiche 'A. Rossi Fanelli', Università di Roma 'La Sapienza', Piazzale Aldo Moro 5, Roma I-00185, Italia. \\ Tel: + 39-06-4450291; Fax: + 39-06-4440062; E-mail: paolo.sarti@uniroma1.it
}

Cell Death and Differentiation (2004) 11, 479-484. doi:10.1038/sj.cdd.4401323

Published online 9 January 2004

Villa Vigoni Conference, Villa Vigoni, Loveno di Menaggio (CO) Italy, 7-9 April 2003

The Italian-German meeting 'Neurodegeneration and energy metabolism: from chemistry to clinics' was hosted last Spring in the wonderful Villa Vigoni, close to the Como lake. Within the tradition of these meetings, about 30 scientists, biochemists and neurologists, deeply dissected the biochemical mechanisms of neurodegeneration and their pathophysiological role, actively exchanging ideas between basic and clinical science. Some particularly hot topics emerged during discussion and are reported here.

\section{Bioenergetic defects and excitotoxicity in the pathogenesis of Parkinson's disease}

Parkinson's disease (PD) is the second most frequent neurodegenerative disorder. The clinical picture of the disease consists of a typical triad of motor symptoms tremor, bradikinesia and rigidity - which impair the ability of parkinsonian patients to execute voluntary movements in an appropriate manner. The pathological substrate of the disease is the progressive loss of dopaminergic neurons of the substantia nigra pars compacta (SNc) projecting to the corpus striatum. SNc and striatum are part of the basal ganglia nuclei whose primary task is to allow the proper execution of voluntary movements. SNc cell loss and resulting dopaminergic denervation of the striatum trigger a cascade of functional changes in the basal ganglia circuitry, ultimately leading to hyperactivity of the subthalamic nucleus (STN) the only excitatory (glutamatergic) nucleus of the circuit which keeps the basal ganglia output nuclei under direct control. ${ }^{1}$ This alteration is now recognized as the neural substrate for PD motor symptoms, but there is also increasing evidence that subthalamic hyperactivity or, more in general, increased glutamatergic tone within the circuit, may contribute to the neurodegenerative process underlying the disease.
Intrinsic high levels of dopamine and iron, above all, expose the SNc to a high rate of reactive oxygen species formation, which translates into potential oxidative damage. In PD, this baseline condition is likely to combine with other cytotoxic mechanisms, such as the impairment of mitochondrial enzyme complex I and/or abnormal protein aggregation, which may result from genetic mutations - particularly in the familial forms of PD - and/or environmental exposures. All these conditions cooperate to trigger the neurodegenerative process and increase the vulnerability of nigral neurons, to further sustain progression of the neuronal damage ${ }^{2}$ by potentially cytotoxic agents that may intervene at later stages. In this context, glutamate may play a central role. It mediates neurotransmission at crucial points of the basal ganglia circuit: within the striatum, for example, glutamate mediates the cortical excitatory input to striatal projection neurons. More importantly, glutamate mediates the excitatory activity of STN projections that, in addition to the basal ganglia output nuclei, target also SNc neurons. ${ }^{1}$ Glutamate can also act as a neurotoxin, mainly through activation of the $\mathrm{N}$-methyl-Daspartate (NMDA) receptor subtype, which may cause cellular calcium influx dysregulation, leading to the cell death of both apoptotic and necrotic type. Due to the peculiar characteristics of the ion channel associated with the NMDA receptor, this phenomenon induces/is enhanced by the presence of a cellular energetic impairment. ${ }^{3}$ Complex I defects associated with PD cause the depletion of ATP levels, rendering the cell more permeable to the glutamate-induced calcium influx. ${ }^{1}$ In PD, the pathological overactivity of subthalamic glutamatergic projections reverberates on the surviving SNc neurons. A self-sustaining vicious cycle may therefore ensue, with the nigrostriatal damage causing subthalamic hyperactivity that, in turn, would sustain further progression of the nigrostriatal damage with an excitotoxic 
mechanism. There is experimental evidence that blocking STN excitatory activity protects SNc neurons against the neurotoxicity of 6-hydroxydopamine (6-OHDA), a toxin extremely selective for dopaminergic neurons. It has recently been found that chronic blockade of STN NMDA receptors with the antagonist dizolcipine (MK-801) reduces the metabolic activity of the STN, and prevents, in the SNc, the cell loss caused by $6-O H D A .{ }^{4}$ Similar, or even greater, protection of nigral neurons against 6-OHDA toxicity is observed when MK801 is given through a systemic, subcutaneous, route of administration (Blandini et al., unpublished data). Significant protection of nigral neurons has also been obtained by inducing a phenotypic shift in STN neurons - from excitatory to inhibitory - using a gene-transfer technique. ${ }^{5}$ In this case, the expression of glutamic acid decarboxylase, the enzyme responsible for the synthesis of inhibitory neurotransmitter GABA, has been induced in STN neurons of rats. The result is that rats, whose STN cells have been transformed into GABAreleasing neurons, are protected against 6-OHDA toxicity. These observations in the rodent model confirm that the interruption of the vicious circle linking nigrostriatal degeneration to glutamatergic overactivity may be essential to neuroprotection in PD.

In conclusion, an interplay between intrinsic bioenergetic defects and secondary glutamatergic hyperactivity targeting surviving SNc neurons takes place in PD. Such a combination is likely to play a major role in the degenerative process, and may represent a potential site of intervention for future neuroprotective strategies.

\section{Endocrine disorders in neurodegeneration}

Neurodegenerative disorders are characterized by a progressive, symmetric loss of cells. Lesions are usually confined to defined brain areas, suggesting that local factors, often under genetic control, determine the selective neuronal susceptibility. A number of studies, however, have shown that peripheral, non-neuronal cells reflect systemic, metabolic disorders, which are likely related to the local pathogenesis, in different neurodegenerative disorders, including Alzheimer's disease (AD).

Particular interest has arisen from the recently recognized association among hyperinsulinemia, diabetes and AD. For many years, it has been known that insulin resistance, or diabetes, is frequently associated with slowly progressive cognitive impairment, and eventually with dementia. As diabetes mellitus is a risk factor for stroke, a widely accepted hypothesis in the past has stressed the role of the vascular dysfunction and of multifocal ischaemic insults in the pathogenesis of cognitive decline. In the last few years, a number of authors reported an association of diabetes with the neuropathological hallmarks of the AD. ${ }^{6}$ The epidemiological data are supported by the evidence that insulin may modulate cognitive activity by a direct effect on the central nervous system, both in humans and laboratory animals.

The mechanisms by which insulin would affect cognition and cause neurodegeneration are uncertain. Insulindegrading enzyme (IDE) is thought to regulate extracellular levels of the beta-amyloid protein, probably by promoting its degradation, an effect that may be dependent on apolipoprotein $E$ genotype. Furthermore, linkage analysis shows an association between genetic markers on chromosome 10q, which map near the IDE gene, and late-onset $A D .^{7}$ Strong evidence for a role of IDE in the pathogenesis of the AD comes from the recent observation that mice with homozygous deletions of the IDE gene show a considerable decrease in degradation of the beta-amyloid protein. ${ }^{8}$ In addition, insulin phosphorylates and activates protein kinase $\mathrm{B}$ - also known as protein kinase Akt - which, in turn, inactivates glycogen synthase kinase- $3 \beta$ (GSK-3 $\beta$ ). GSK- $3 \beta$ is involved in a wide range of cellular processes potentially related to neurodegeneration. In particular, GSK-3 $\beta$ phosphorylates tau proteins, a significant step in the pathogenesis of the AD. ${ }^{9}$

It is, therefore, a substantial hypothesis that the neurodegeneration associated with the $A D$ is causally related to an impairment of the intracellular signalling linked to the insulin receptor. This hypothesis was tested in collaboration with Ferdinando Nicoletti (Department of Pharmacology, University of Roma 'La Sapienza'). The study was carried out in lymphocytes, isolated from blood withdrawn from subjects with mild to moderate cognitive impairment due to probable $\mathrm{AD}$, or from matched controls. Lymphocytes were stimulated in vitro with insulin, and phosphorylation of Akt was found impaired in the AD subjects, as compared to controls.

\section{Calcium-binding proteins in Alzheimer's disease: the good or the bad guys?}

$A D$ is mainly characterized by a massive loss of neurons in the final stages of illness. However, already at early stages, an impairment of memory and cognition can be observed. Both phenomena correlate with the appearance of amyloid plaques and neurofibrillary tangles, the pathological hallmarks of the disease, in key brain structures for cognition. A striking feature of pathophysiology in $A D$ seems to be a changed calcium homeostasis. A calcium overload eventually leads to activation of several processes of necrotic and apoptotic cell death. The observed disturbances in calcium signalling imply the involvement of calcium-binding proteins as either direct or indirect mediators of pathophysiological changes in the course of the disease. The likely candidates are members of the superfamily of EF-hand calcium-binding proteins, that is, the calcium buffer and calcium sensor proteins. An involvement of the calcium buffer proteins parvalbumin and calbindinD28K in neurodegenerative processes has been postulated. ${ }^{10}$ Both proteins have a neuroprotective role and show a reduced expression level in $A D$ brains. An increased expression level of calbindin-D28K was shown to correlate with reduced calcium-induced cell death, in hippocampal cell cultures and in the hippocampus in vivo. ${ }^{11}$ Besides calcium buffer proteins, the neuronal calcium sensor (NCS) proteins are also implicated in AD. The immunoreactivity for the visininlike proteins (VILIPS), namely VILIP-1 and -3 , is reduced in Alzheimer brains in comparison to control brains. ${ }^{12}$ The number and staining intensity of VILIP-1- and -3-immunoreactive neurons is reduced in the temporal and entorhinal cortex, and, interestingly, immunoreactivity can be detected in close association with amyloid plaques and neurofibrillary 
tangles. Accordingly, for VILIP-1, Western blots revealed significantly reduced protein levels in tissue extracts of the temporal cortex of AD patients. ${ }^{12}$ Morphological and neurochemical findings, therefore, point to a higher vulnerability of VILIP-1-expressing neurons in AD. In contrast, cortical neurons that contain calcium-buffering proteins, such as calbindin-D28K, appear to be relatively protected against neurodegeneration. ${ }^{10}$

Indeed, this assumption was confirmed in cytotoxicity assays in PC12 model neurons transfected with the calcium sensor VILIP-1 and/or the calcium buffer calbindin-D28K. Whereas VILIP-1 expression enhanced the neurotoxic effect of the calcium ionophor ionomycin, calbindin-D28K protected against ionomycin-induced cytotoxicity. ${ }^{12}$ In addition, calbindin-D28K rescued VILIP-1-mediated cytotoxicity at low ionomycin concentrations. Since VILIP-1 was found to be associated with fibrillary tangles in $A D$ brains, it was tested whether there is a functional link between VILIP-1 and tau hyperphosphorylation. VILIP-1 expression enhanced the phosphorylation of tau protein compared to nontransfected cells. Again, this VILIP-1 effect was attenuated via coexpression with the calcium buffer protein calbindin-D28K ${ }^{13}$ (Figure 1, Top, neuron).

In summary, the calcium sensor protein may influence tau phosphorylation and play a role in calcium-mediated neurotoxicity in contrast to the previously discovered protective effect of calcium buffer proteins. The combination of expression of calcium buffer and/or calcium sensor proteins may decide whether pathological fibrillary tangles are formed and whether neurons are particularly vulnerable to, or protected against, pathologically induced calcium concentrations.

Finally, since calcium buffer and sensor proteins have been directly implicated in learning and memory processes, ${ }^{14,15} \mathrm{a}$ link can be envisaged between their role in plasticity and in neurodegeneration (see the following section and Figure $1 \mathrm{a}, \mathrm{b})$. Thus, the reduction in protein expression may also correlate with the pathophysiological disturbances leading to the early cognitive impairments in AD.

\section{Metabotropic glutamate receptors as mediators of the plasticity-pathology continuum}

The hippocampus is a key structure for learning and memory formation in the mammalian brain. It is particularly affected in neurodegenerative disorders such as AD. Information storage in the hippocampus is believed to necessitate persistent, longlasting alterations in the efficacy of synaptic communication (synaptic plasticity). These alterations are expressed in the form of long-term potentiation (LTP) or depression (LTD) of synaptic efficacy, which arise as a consequence of patterned electrical activation of afferent fibres. ${ }^{16}$ Both LTP and LTD critically require the activation of metabotropic glutamate receptors (mGluRs) for persistent expression to occur: whereas antagonists of group I mGluRs partially impair the expression of LTD, antagonists of group II mGluRs completely inhibit the expression. ${ }^{17}$ On the other hand, group II antagonists do not affect the expression of LTP, whereas activation of group 1 mGluRs is critically required. ${ }^{17}$ Thus, mGluRs may function as bidirectional regulators of
Neuron

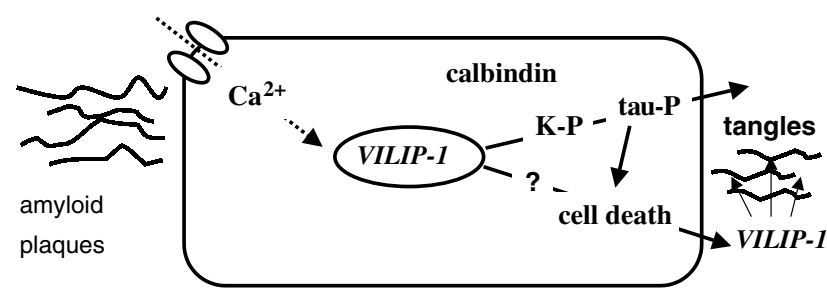

Synapses

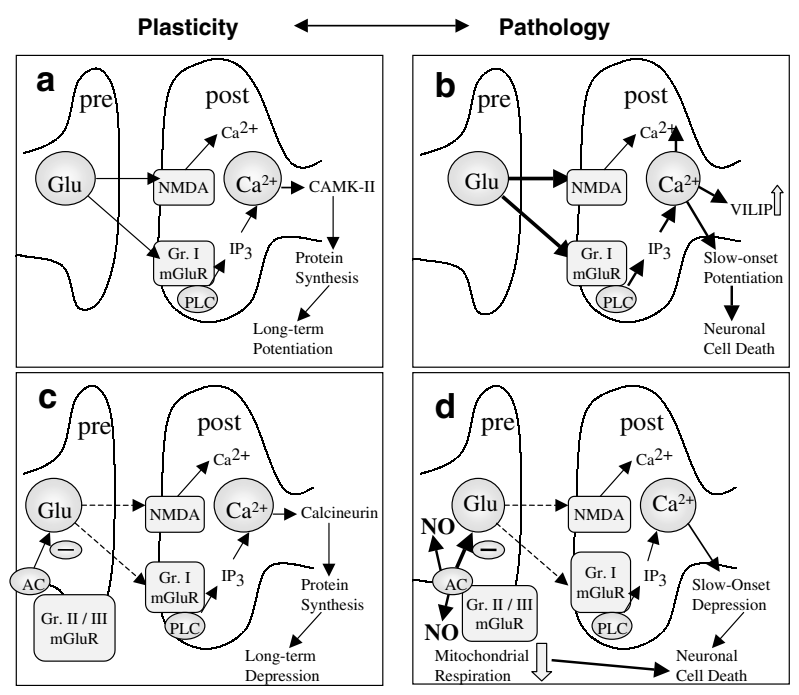

Figure 1 (Top, neuron) Model for the involvement of the calcium buffer (calbindin-D28K) and the calcium sensor protein (VILIP-1) in the pathophysiology of $A D$. In neurons, the pathological increase of calcium levels leads to calcium sensor (VILIP-1)-mediated neurotoxicity via yet unknown mechanisms (indicated via influencing kinases or phosphatases ( $k-p)$, probably as a ?), attenuated by calbindin. VILIP-1 promotes tau hyperphosphorylation, probably linking amyloid and tau pathology. Following cell death, VILIP-1 is released from the dead neurons and decorates extracellular tangles and amyloid plaques. (Bottom, synapses) Physiological and pathological mechanisms triggered by metabotropic glutamate receptors. (a) Under physiological conditions, release of glutamate by high-frequency patterned electrical stimulation of afferent fibres leads in sequence to the activation of postsynaptic group I mGluRs, phospholipase C $(\mathrm{PLC})$ with the production of inositol trisphosphate $\left(\mathrm{IP}_{3}\right)$, and calcium release from intracellular calcium stores. Calcium elevation, together with calcium entry via the glutamate activation of ionotropic NMDA receptors, leads to stimulation of calcium-calmodulin kinase II (CAMKII) and ultimately to protein synthesis, which enables persistent expression of LTP. (b) Under pathological conditions, intense activation of, for example, group I mGluRs (broader arrows) leads to an exorbitant release of calcium from intracellular stores, induction of VILIP $(\Uparrow)$ and the disturbance of intracellular calcium homeostasis. The initial consequence of the strong calcium elevation is slow-onset potentiation; this is succeeded by neuronal cell death. (c) Under physiological conditions, release of glutamate by low-frequency patterned electrical stimulation of afferent fibres leads to weak activation of group I mGluRs and stimulation of presynaptic group II and III mGluRs. Activation of the latter serves to reduce presynaptic glutamate release. The relatively lower postsynaptic calcium elevation leads to stimulation of calcineurin and a phosphate-dependent cascade which triggers protein synthesis relevant for long-term persistence of LTD. (d) The pathological activation of group II and III mGluRs leads to strong inhibition of adenylyl cyclase (AC), which in turn causes strong inhibition of glutamate release, potent activation of NO and inhibition of mitochondrial respiration $(\Downarrow)$. The initial consequence of the suppressed glutamate release is slow-onset depression. Long-term effects comprise neuronal cell death 
hippocampal synaptic plasticity. Interestingly, mGluRs are able to induce synaptic plasticity independent of patterned electrical stimulation. Whereas weak activation of group I mGluRs coupled with patterned electrical stimulation leads to induction of LTP (Figure 1b) and LTD (Figure 1c), application of agonists of group I mGluRs (independent of patterned electrical stimulation) dose-dependently leads to slow-onset potentiation in the hippocampus. ${ }^{18}$ Agonists of group II and III mGluRs produce slow-onset depression. Both phenomena are associated with pathological consequences for hippocampal cell viability and function. The possibility thus arises that mGluRs are not just critical factors in the induction of hippocampal synaptic plasticity, but may also contribute significantly to hippocampal pathology and neurodegeneration.

Metabotropic glutamate receptors (mGluRs) comprise at least eight subtypes (1-8), which are classified into three distinct subgroups, named I, II and III mGluRs, respectively, ${ }^{19}$ based on their biochemical coupling and agonist preference. The observation that the pharmacological activation of phospholipase C-coupled group I mGluRs elicits slow-onset potentiation in vivo led to speculation that this phenomenon could be linked to excessive calcium release from intracellular stores. Histological analysis revealed that slow-onset potentiation caused chronic loss of cell viability and density in the rat hippocampus, suggesting that an overactivation of group I mGluRs could lead to a malfunction of intracellular calciumbuffering mechanisms (Figure 1b, c). Accordingly, slow-onset potentiation was also associated with an increased expression of the EF-hand calcium-binding protein VILIP-1. ${ }^{15}$ Interestingly, VILIP is associated with the pathological hallmarks of $A D$ and leads to increased neurotoxicity in cell culture systems. ${ }^{13}$ Taken together, these results suggest that overactivation of group I mGluRs may lead to a disruption of intracellular calcium homeostasis and, consequently, to cell death, which arises subsequent to the induction of slow-onset potentiation.

Slow-onset depression occurs following activation of group III mGluRs. ${ }^{20}$ Neuroprotective effects of L-2-amino-4-phosphonobutyric acid (AP4) with regard to neurodegenerative events such as anoxia have been reported. ${ }^{21}$ It was thus surprising to discover that slow-onset depression elicits a loss of cell viability and density in the hippocampus in vivo (Manahan-Vaughan et al., unpublished data). Under physiological conditions, group III mGluRs are negatively coupled to adenylyl cyclase. ${ }^{22}$ However, under conditions of cell endangerment, AP4 appears to stimulate adenylyl cyclase production, which in turn inhibits the neurotoxic effects of free radicals such as nitric oxide ${ }^{21}$ (Figure $1 b, d$ ). In contrast, the strong inhibition of adenylyl cyclase necessary to chemically induce synaptic depression may cause overstimulation of nitric oxide production, with a strong depression of mitochondrial respiration and impoverishment of cell viability and apoptosis in the hippocampus. ${ }^{23}$ The finding that mGluRs contribute to both synaptic plasticity and pathology qualify them as likely candidates for regulators of a plasticitypathology continuum (Table 1). Thus, under circumstances where mGluRs are physiologically activated, they serve to support mechanisms underlying the expression of synaptic plasticity, whereas under conditions of pathological activation,
Table 1 The metabotropic glutamate receptor, as mediator of the plasticity pathology continuum

\begin{tabular}{lll}
\hline & $\begin{array}{l}\text { Physiological } \\
\text { activation }\end{array}$ & $\begin{array}{l}\text { Pathological } \\
\text { activation }\end{array}$ \\
\hline Group I mGluR & LTP facilitation & $\begin{array}{l}\text { Slow-onset potentiation } \\
\text { Cell death }\end{array}$ \\
Group II mGluR & LTD facilitation & $\begin{array}{l}\text { Slow-onset depression } \\
\text { (neurotoxicity unknown) }\end{array}$ \\
Group III mGluR & $\begin{array}{l}\text { LTP inhibition } \\
\text { LTD facilitation } \\
\text { LTP inhibition }\end{array}$ & $\begin{array}{l}\text { Slow-onset depression } \\
\text { Cell death }\end{array}$ \\
\hline
\end{tabular}

Pharmacological activation of mGluRs using agonist concentrations which do not alter basal synaptic transmission can convert short-term plasticity into longterm plasticity in the hippocampus in vivo. Whereas agonists of group I mGluRs convert short-term potentiation into LTP, agonists of group II and III mGluRs facilitate LTD and inhibit LTP in vivo. Agonist concentrations which lead to induction of chemically induced synaptic plasticity result in cell death regardless of whether slow-onset potentiation or slow-onset depression was elicited ${ }^{18,20,24}$

they can mediate mechanisms of neurotoxicity and neurodegeneration in neurones. ${ }^{24}$ This may occur, for example, during the intense and prolonged glutamate release elicited during an epileptic seizure, or following neuronal malfunction, as in AD.

\section{Neurodegeneration, nitric oxide (NO) and mitochondria}

The balance between nitric oxide (NO) and the superoxide ion $\left(\mathrm{O}_{2}^{-}\right)$is of paramount importance for cell signalling and survival. ${ }^{25,26}$ Concentrations of these species exceeding the physiological concentrations may trigger pathological responses, leading to oxidative/nitrosative stress. This is likely the case in all cerebrovascular pathologies accompanied with cerebral ischaemia, leading to glutamate-dependent overactivation of the NMDA receptor, and stimulation of the calcium-dependent NO synthase (NOS) with persistent release of NO.,27 Similar events have been proposed to occur in PD, multiple sclerosis, amyotrophic lateral sclerosis and Huntington disease. In the nervous system, the NO chemistry has proved responsible for positive or negative effects, depending on experimental conditions. Several years of investigation have suggested that the electronic configuration of the species $\mathrm{NO}^{-}$, $\mathrm{NO}^{+}$and $\mathrm{NO}^{-}$, as well as their actual concentration in a given cell compartment, can make the difference. Since clear information about a differential behaviour in the mitochondria of the aforementioned species is not available, attention is limited here to NO, that is, to radical nitric oxide. A first conclusion is that free $\mathrm{NO}$, persistent in the cell environment above a concentration value still not defined, may become toxic: this occurs when the NO buffering/degrading capacity is saturated, and/or the rate of NO synthesis and degradation is not balanced. ${ }^{28}$ While the knowledge of the pathways leading to NO synthesis and buffering has grown rapidly, very little is known about the reactions involved in NO degradation. Evaluation of interactions between NO and mitochondria suggests that a tight crosstalk exists between the respiratory chain and NO. Mitochondrial function is controlled by NO at different levels. ${ }^{29}$ If persisting in the environment, $\mathrm{NO}$ induces impairment of ATP synthesis, the opening of the membrane transition pore, 
with apoptosis and eventually cell death. ${ }^{29}$ This is not surprising, as NO inhibits several mitochondrial metalloproteins very efficiently. Particularly, NO reacts with and controls the electron-entry gate of the respiratory chain, that is, complex I, as well as the terminal site of the chain, that is, complex IV (the cytochrome $c$ oxidase, CcOX). Complex IV reacts with $\mathrm{NO}$ on a much shorter timescale than complex I, milliseconds versus hours, and at physiological NO concentrations, that is, $\mathrm{nM}$ versus $\mathrm{mM} .{ }^{28} \mathrm{NO}$ inhibits $\mathrm{CcOX}$ promptly and even in the presence of oxygen, and inhibition is maintained until free $\mathrm{NO}$ is available in the mitochondrion. Based on these simple considerations, sustained by a wealth of experimental data, upon exposure of mitochondria to NO, any acute phenomenon observed, such as inhibition of respiration, should take in to account the interaction of NO with complex IV, whereas more chronic phenomena may include all the other mitochondrial targets. Indeed, owing to the potential toxicity of $\mathrm{NO}$, its concentration is kept under strict control by the cell. The NO scavenger glutathione efficiently modulates the bioavailability of NO. Myoglobin in myocytes, $^{30}$ neuroglobin and cytoglobin in neurons are intracellular metalloproteins endowed with a hexa-coordinated haemeiron, and may play a similar NO-scavenging role. ${ }^{31}$ Within this frame, it would be expected that $\mathrm{NO}$ and the superoxide ion $\mathrm{O}_{2}^{-}$rise during ischaemia, the former since needed as a vasodilator, the latter due to the cell/mitochondria anaerobicity! Both radicals are potentially toxic; they react in a $1: 1$ stoichiometry to form, at a diffusion-limited rate, the peroxynitrite $\left(\mathrm{ONOO}^{-}\right)$; this is a species still highly reactive and dangerous for the cell, as are the original unreacted radicals.

In contrast with the clear toxic effect of NO on mitochondria, the presence of a mitochondrial NOS $^{32}$ seems to suggest that NO may play a physiological role by inhibiting a fraction of $\mathrm{CcOX}$, thus making the respiratory chain sensitive to oxygen, exactly in the cell/tissue $\mathrm{O}_{2}$ concentration range $(5-20 \mu \mathrm{M}){ }^{33}$ This fully reversible inhibition might be useful, allowing a deeper $\mathrm{O}_{2}$ permeation within tissues, and its utilization by cells more distant from capillaries. ${ }^{34}$ In this perspective, it is crucial to know how the NO-inhibited $\mathrm{CcOX}$ molecules recover function. It is evident that, for $\mathrm{NO}$ to be a $\mathrm{CcOX}$ modulator, the binding reaction should be fast, but the inhibition also should be promptly released upon removal of free NO from the mitochondrion. This has been shown to occur, and with some interesting additional features, bound to the mechanism followed during the reaction between $\mathrm{NO}$ and $\mathrm{CcOX}{ }^{28}$ Depending on a number of parameters, including the level of electron flow rate through the respiratory chain, two alternative mechanisms of reaction have been described in detail. One mechanism leads to accumulation of the nitrite $\mathrm{CcOX}-\mathrm{NO}_{2}^{-}$adduct, the other forms the nitrosyl CcOX-NO adduct:

$\begin{array}{clcll}\text { reactants } & & \text { inhibited adducts } & & \text { enzyme regeneration } \\ \mathrm{CcOX}+\mathrm{NO} & \rightarrow & \mathrm{CcOX}-\mathrm{NO}_{2}^{-} & \rightarrow & \mathrm{CcOX}+\mathrm{NO}_{2}^{-} \text {(rapid) } \\ \mathrm{CcOX}+\mathrm{NO} \rightarrow \mathrm{CcOX}-\mathrm{NO} & \rightarrow & \mathrm{CcOX}+\mathrm{NO} \text { (slow) }\end{array}$

Both adducts are inhibited, but regenerate fully active CcOX with different kinetics; the nitrite adduct much more rapidly than the nitrosyl one and the latter releasing NO, still active. Interestingly, very recent results ${ }^{35}$ showed that neuroblastoma cells grown in culture favour the apparently safer nitrite

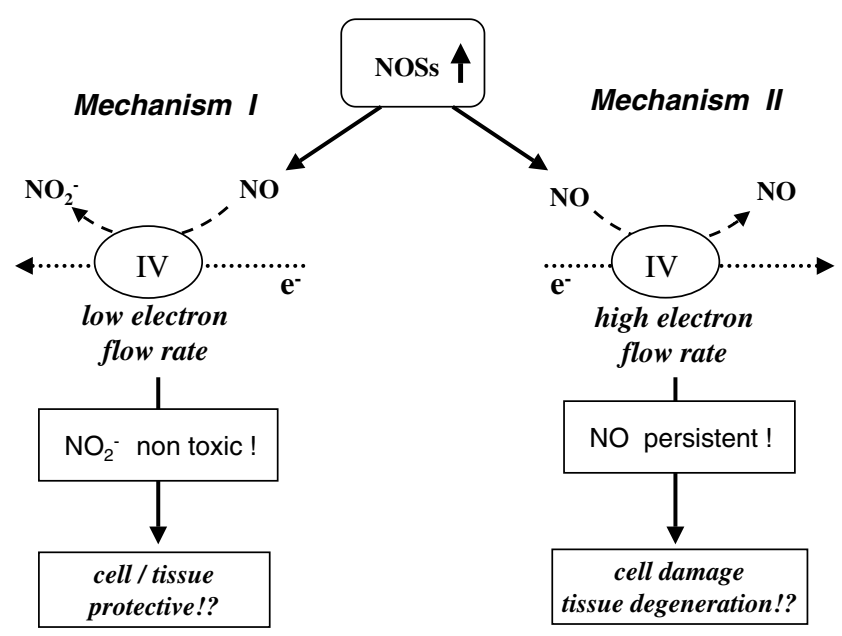

Figure 2 Differential effects of NO on mitochondrial respiration induced by the reaction with complex IV. NO, produced by stimulated NOSs $(\uparrow)$, reacts with complex IV following two mechanisms, depending on the level of electron flow through the chain, low (left) or high (right), leading to products with different pathophysiological implications

mechanism. It remains to be investigated if other cells and tissues in our body share this strategy or whether this is a peculiarity of cultured (tumour) cells. A consequence of the two-mechanism model is that those mitochondrial metabolic conditions favouring $\mathrm{CcOX}$ nitrosylation would induce a respiratory failure that, if persistent, would most likely be detrimental for the cell (Figure 2).

\section{Concluding remarks}

Neurodegeneration underlies a wide variety of neurological disorders, whose genetic, metabolic and clinical features appear definitely heterogeneous, at least at first sight. This notwithstanding, some biochemical features can be identified as common to most neurodegenerative states. For example, a pivotal role in the neuronal damage seems to be played by changes in the calcium fluxes and trafficking. Calcium movements control neural plasticity mechanisms and probably, based on most recent evidence, the aerobic mitochondrial metabolism via activation of constitutive NOS and NO release. Various mechanisms may be involved in this phenomenon, such as overstimulation/altered responsiveness of glutamate receptors of both the ionotropic and metabotropic subclasses, and/or modifications of calciumbinding proteins. The increased tendency of neurons to undergo apoptosis, in particular, seems also a common feature in neurodegeneration. This finding is fully consistent with an excessive calcium influx or failure of the calciumbuffering/-sensing systems, always exposing the cell to apoptotic or necrotic risk. Finally, it is worth keeping in mind that reactive oxygen and nitrogen species - ROS and RNS respectively - particularly NO and peroxynitrite - have been shown to be responsible for inactivation of the respiratory complexes, as well as membrane peroxidation. The ultimate irreversible activation of the membrane permeability transition pore and cell death could be easily explained on this basis. 


\section{Acknowledgements}

The meeting was generously supported by the Fondazione SIGMA-TAU (Pomezia, RM, Italy). This work was supported by Ministero dell'Istruzione, dell'Università e della Ricerca (MIUR) of Italy - PRIN 'Bioenergetica: aspetti genetici, biochimici e fisiopatologici', as well as by the Deutsche Forschungsgemeinscahft grant, Ma 1843, to DMV.

1. Blandini $F$ et al. (2000) Prog. Neurobiol. 62: 63-88

2. Betarbet $R$ et al. (2002) Brain Pathol. 12: 499-510

3. Sarti $P$ et al. (1999) FASEB J. 13: 191-197

4. Blandini F et al. (2001) Ann. Neurol. 49: 525-529

5. Luo J et al. (2002) Science 298: 425-429

6. Ott A et al. (1996) Diabetologia 39: 1392-1397

7. Bertram L et al. (2000) Science 290: 2302-2303

8. Farris W et al. (2003) Proc. Natl. Acad. Sci. USA 100: 4162-4167

9. Kaytor MD and Orr HT (2002) Curr. Opin. Neurobiol. 12: 275-278

10. Heizmann CW and Braun K (1992) Trends Neurosci. 15: 259-264

11. Phillips RG et al. (1999) J. Neurochem. 73: 1200-1205

12. Braunewell KH et al. (2001) Dement. Geriatr. Cogn. Disord. 12: 110-116

13. Schnurra I et al. (2001) Neurobiol. Dis. 8: 900-909

14. Gomez M et al. (2001) Neuron 30: 241-248

15. Braunewell KH et al. (2003) Neuropharmacology 44: 707-715
16. Bear MF (1996) Proc. Natl. Acad. Sci. USA 93: 13453-13459

17. Manahan-Vaughan D (1997) J. Neurosci. 17: 3303-3311

18. Manahan-Vaughan D and Reymann KG (1995) Eur. J. Pharmacol. 294: 497-503

19. Nakanishi S et al. (1998) Brain Res. Rev. 26: 230-235

20. Manahan-Vaughan D and Reymann KG (1995) Neuropharmacology 34 991-1001

21. Maiese K et al. (1996) J. Neurochem. 66: 2419-2428

22. Thomsen C (1997) Gen. Pharmacol. 29: 151-158

23. Thomson M (2002) Cell Mol. Life Sci. 59: 213-219

24. Braunewell KH and Manahan-Vaughan D (2003) Rev. Neurosci. 12: $121-140$

25. Sarti $P$ et al. (2002) Cell Death Differ. 9: 1160-1162

26. Ischiropoulos H and Beckman JS (2003) J. Clin. Invest. 111: 163-169

27. Law A et al. (2001) Brain Res. Rev. 35: 73-96

28. Sarti $P$ et al. (2003) Free Radic. Biol. Med. 34: 509-520

29. Moncada S and Erusalimsky JD (2003) Nat. Rev. Mol. Cell Biol. 3: 214-220

30. Brunori M (2001) Trends Biochem. Sci. 26: 209-210

31. Pesce A et al. (2002) EMBO Rep. 3: 1146-1151

32. Giulivi C (2003) Free Radic. Biol. Med. 34: 397-408

33. Brown GC (1995) FEBS Lett. 369: 136-139

34. Brunori M et al. (2003) Biochim. Biophys. Acta (in press)

35. Mastronicola D et al. (2003) Cell. Mol. Life Sci. 60: 1752-1759 